\title{
Built environment determinants of bicycle volume: A longitudinal analysis
}

\author{
Peng Chen \\ Harbin Institute of Technology \\ Shenzhen Campus \\ chenp5@uw.edu \\ Feiyang Sun \\ University of Washington \\ fs377@uw.edu
}

\author{
Jiangping Zhou \\ University of Hong Kong \\ zhoujp@hku.hk
}

\begin{abstract}
This study examines determinants of bicycle volume in the built environment with a five-year bicycle count dataset from Seattle, Washington. A generalized linear mixed model (GLMM) is used to capture the bicycle volume change over time while controlling for temporal autocorrelations. The GLMM assumes that bicycle count follows a Poisson distribution. The model results show that (1) the variables of non-winter seasons, peak hours, and weekends are positively associated with the increase of bicycle counts over time; (2) bicycle counts are fewer in steep areas; (3) bicycle counts are greater in zones with more mixed land use, a higher percentage of water bodies, or a greater percentage of workplaces; (4) the increment of bicycle infrastructure is positively associated with the increase of bicycle volume; and (5) bicycling is more popular in neighborhoods with a greater percentage of whites and younger adults. It concludes that areas with a smaller slope variation, a higher employment density, and a shorter distance to water bodies encourage bicycling. This conclusion suggests that to best boost bicycling, decision-makers should consider building more bicycle facilities in flat areas and integrating the facilities with employment densification and open-space creation and planning.
\end{abstract}

Keywords: bicycle volume, built environment, longitudinal data analysis, generalized linear mixed model

\section{Introduction}

Bicycling is a sustainable alternative to driving for short-distance trips because of its economical, healthy, and environmentally-friendly attributes. The number of bicyclists has been growing in many large United States (US) cities during the last decade. Yet, data capturing the use of bicycle tracks and

Copyright 2017 Peng Chen, Jiangping Zhou \& Feiyang Sun http://dx.doi.org/10.5198/jtlu.2017.892

ISSN: 1938-7849 | Licensed under the Creative Commons Attribution - Noncommercial License 3.0

The Journal of Transport and Land Use is the official journal of the World Society for Transport and Land Use (WSTLUR) and is published and sponsored by the University of Minnesota Center for Transportation Studies.

\section{Article history:}

Received: February 11, 2017

Received in revised form:

January 23, 2017

Accepted: March 18, 2017

Available online: June 13, 2017 
bike lanes are generally unavailable, which significantly affects the quality of existing bicycle research (Hankey et al., 2012). Bicycling only accounts for $1 \%$ of all trips in the US. No monitoring network has been established for bicycle usage (Hankey, Lindsey, \& Marshall, 2014), and the mass installment of bicycle counters at road intersections is considered a cost-ineffective infrastructure investment (El Esawey, Mosa, \& Nasr, 2015).

To fill in the gap between the need for bicycle volume data and the lack of measuring devices, some local authorities employ surveys to approximate bicycle counts. The bicycle volume is estimated by surveying regional household travel behaviors and counting the number of bicycle trips. However, the survey-reported bicycle trips are zonal-based counts. Thus, the geographical unit of the data is too large to predict the bicycle volume at road intersections, and misses short bicycle trips in a zone in many cases. Furthermore, the survey data are mostly collected from bicycle trips for commuting purposes. In the city of Seattle, two-thirds of the bicycle trips are used for general recreation and exercise (Seattle Department of Transportation, 2014). The majority of bicycle trips for other purposes is thus overlooked. In addition, the survey data cannot reflect the real-time changes of bicycle counts, which constrains the understanding of many time-varying determinants of bike volume.

The bicycle volume data play an important role in policy making, bicycle route planning, road safety improvement, municipal investment, and maintenance priorities (Hankey et al., 2014). But all these benefits must be based on ample and accurate bicycle volume data. The absence of bicycle volume data results in an ineffective evaluation of the adaptability and capability for a safe and attractive bicycling environment (Bhatia \& Wier, 2011). Only with more bicycle volume measurements can bicycle infrastructure investment plans prioritize areas of a high bicycle volume, and therefore better utilize limited budgets assigned to bicycle programs. Most importantly, bicycle volume data can help forecast bicycle crash risk, which in turn inform road-safety improvements. Bicycle crash risk is often calculated by the number of bicycle collisions divided by a denominator indicating bike volume such as the number of bicycle trips, the bicycle miles traveled, or the number of bicyclists (Vanparijs, Panis, Meeusen, \& de Geus, 2015). Generating these preferred denominators, mostly unavailable now, will help researchers accurately measure the bicycle crash risk.

Realizing the importance of measuring bicycle volume, several US cities, such as Portland, Minneapolis, and Seattle, have started manually and automated counting bicycles. These new datasets provide researchers with opportunities to identify explanatory factors, especially time-varying factors, of bicycle volume to propose more pertinent policies to promote bicycling.

The present study takes the advantage of the longitudinal bike counts and other related datasets in Seattle to investigate a key research question: by adjusting the effects of winter/non-winter seasons, peak hours, and weekends, what built environment features are associated with bicycle counts? The incorporation of time-varying variables not only presents an alternative modeling approach to address temporal autocorrelations but also enriches the policy discussion in promoting bicycling in the US.

This study is organized as follows: section two provides a review of the existing studies on bicycle volume and bicycle use; section three presents the research design including data description, statistical concerns and model specification, and variable selection; section four discusses the results of descriptive and inferential analyses; section five synthesizes findings, discusses their policy implications, and directs future research.

\section{Literature review}

\subsection{Studies with bicycle volume data}

Most local authorities do not count bicycles in the US. Consequently, the existing research on bicycle 
volume is far from adequate. With limited bicycle count data, several previous studies correlated bicycle volume with built environment factors, using Minneapolis and Alameda County for empirical analyses (Griswold, Medury, \& Schneider, 2011; Hankey \& Lindsey, 2016; Hankey et al., 2012). With similar research objectives, another study examined the relationship between built environment features and public bicycle volume (Faghih-Imani, Eluru, El-Geneidy, Rabbat, \& Haq, 2014). Those studies found that the mixture of land use and the density of commercial facilities were positively associated with bicycle volume (Griswold et al., 2011; Hankey et al., 2012). In addition, hilliness was a negative predictor of bicycle volume (Griswold et al., 2011).

Several other studies predicted bicycle volume without a focus on the built environment. For instance, the effect of seasonal changes on bicycle counts was examined via an autoregressive integrated moving average model using automatically counted bicycle data from Vancouver, Canada, where the effect of built environment features was not adjusted (Gallop, Tse, \& Zhao, 2012). Other studies were more interested in forecasting bicycle volume via simulations that adjusted for the effects of weather, peak-hours, and seasons (El Esawey, Lim, Sayed, \& Mosa, 2013; El Esawey et al., 2015; Gosse \& Clarens, 2014; Tin, Woodward, Robinson, \& Ameratunga, 2012). For instance, El Esawey et al. examined how to impute missing data for consecutive bicycle counts when bicycle counters were malfunctioning (El Esawey et al., 2015) .

In sum, the existing studies using bicycle volume data have identified a set of built environment determinants. However, many of them have not examined a full range of potential determinants, especially time-varying factors, largely due to data limitations. It is necessary to revisit a broader area of research on the factors impacting bicycle use to establish a more comprehensive framework.

\subsection{Factors impacting bicycle use}

"Bicycle use" is a broad term, which can be measured by travel mode choice of bicycling, bicycle volume, bicycle frequency, bicycle miles traveled, and bicycle hours traveled. A wide range of factors are identified as determinants of bicycle use in the current literature (Strauss \& Miranda-Moreno, 2013). This section provides a review by synthesizing the effects of the built environment, transportation policies, socio-demographics, and time-varying factors on bicycle use.

Many existing bicycle studies focus on identifying built environment factors that affect the bicycle use. These studies have discussed ways to encourage more bicycle use, such as building separated cycle tracks (Buehler \& Pucher, 2012; Thomas \& DeRobertis, 2013; Winters, Davidson, Kao, \& Teschke, 2011), launching public bike sharing programs (Heinen, van Wee, \& Maat, 2010; Shaheen, Guzman, $\&$ Zhang, 2010), promoting integration with public transit (Bachand-Marleau, Larsen, \& El-Geneidy, 2011), and reducing vehicle parking in city centers (Shoup, 2005).

Built environment factors associated with bicycle use can be categorized as functional, safety, and aesthetic factors. Trip distance is a core factor affecting bicycle use. A longer trip distance is associated with a greater generalized travel cost resulting from increased travel time (Heinen et al., 2010). Trip distance correlates with city sizes and a number of functional built environment factors, such as density, mixed land use, road connectivity, block size, and destination accessibility (Heinen et al., 2010; Krizek, El-Geneidy, \& Thompson, 2007; Moran, Plaut, \& Epel, 2015; Muhs \& Clifton, 2016; Saelens, Sallis, \& Frank, 2003; Van Acker, Derudder, \& Witlox, 2013) . A compact urban environment—higher density, greater mixed land use, better road connectivity, denser blocks, and closer proximity to destination - can help reduce trip distance, which is more suitable for non-motorized transportation modes (Heinen et al., 2010; Moudon et al., 2005; Saelens et al., 2003). Though the aforementioned factors have been frequently discussed, other essential factors influencing bicycle use, such as the presence of parks, are still underestimated in conventional research frameworks (Heinen et al., 2010). 
Among safety-related built environment factors, the layout of the road network and the provision of bicycle facilities are essential in encouraging bicycle use (Wardman, Tight, \& Page, 2007; Winters et al., 2011) because they both can reduce the perceived risk and conflicts between motorists and bicyclists. Road network layouts affect driving speeds and traffic volume. Specifically, a denser road network allows lower driving speeds and a higher traffic volume (Heinen et al., 2010). Bicyclists commonly share roads with motorists in US cities. As a result, building cycle tracks and isolated bike lanes to separate bicyclists from vehicle traffic creates safety benefits and encourages biking (Reynolds, Harris, Teschke, Cripton, \& Winters, 2009). In a compact developed urban environment with a higher density and more mixed land use, the bicycling environment is safer due to traffic speed reduction (Chen, 2015; Chen \& Shen, 2016).

Aside from functional and safety factors, aesthetical factors play an essential role in bicycle trip generation. Researchers hitherto have not often treated these factors of the built environment, partly because such aesthetic features are relatively hard to quantify. Yet, in a handful of existing studies, scenic routes are found to be preferred by bicyclists (Pikora, Giles-Corti, Bull, Jamrozik, \& Rob, 2003; Stefansdottir, 2014; Su et al., 2014). The majority of bicycle trips is motivated by recreational purposes (Goetzke \& Rave, 2010; Su et al., 2014), and the attractiveness of the built environment acts as a key factor in bicycle routing (Lovasi et al., 2013; Stefansdottir, 2014).

Transportation policies affect the generalized cost of different transportation modes. Economicincentive-based policies — gas taxes, tolling, license auction, parking fees, and congestion pricing- discourage driving, and thus indirectly encourage bicycling. For instance, the cost of driving is high in Germany because of several restrictive policies. The difference between Germany and the US in car ownership and mode choice is largely explained by transportation policies rather than built environment factors (Buehler, Pucher, Merom, \& Bauman, 2011). Bicycling is less likely to be popular in cities with low costs of car purchase, parking, insurance, and gas.

Bicycle use is associated with many socio-demographic factors including gender, age, physical condition, family composition, and occupation (Akar \& Clifton, 2009; Ma \& Dill, 2015; Wang, Lindsey, Schoner, \& Harrison, 2015). Females, senior citizens, and businessmen are less likely to ride bicycles. Socio-economically advantaged people, who are defined by income, employment status, and education, are more likely to participate in active transportation because of their greater awareness of maintaining health (Goodman, Sahlqvist, Ogilvie, \& consortium, 2013; Ma \& Dill, 2015). The presence of children greatly discourages the propensity of biking (Ma \& Dill, 2015).

In terms of individual comfort-related factors, the presence of other amenities, such as showers at trip destinations, bike racks for secure parking, and accessible public bike stations, is correlated with more bicycle use (Buehler, 2012). In addition to the above-mentioned factors, time-varying factors, such as weather, season, peak travel hours, and weekends, are significant factors affecting bicycle use (Faghih-Imani et al., 2014; Heinen et al., 2010). The consideration on factors of the built environment, transportation policies, socio-demographics, individual comfort, and time plays an important role in encouraging bicycle use, which is critical to the success of a bicycle master plan.

\subsection{Bicycle plan implementation and bicycle use}

The studies reviewed above have mostly examined factors associated with bicycle use in general. Only a small number of them have evaluated more specifically the effort of bicycle plans. According to them, it is evident that the establishment of an extensive bicycle network is associated with a high degree of bicycling popularity (dell'Olio, Ibeas, Bordagaray, \& Ortúzar, 2013; Handy, Xing, \& Buehler, 2010; Pucher, Dill, \& Handy, 2010; Schoner \& Levinson, 2014). However, some recent ex-ante and ex-post studies suggest more complicated and contradictory findings. For example, in Portland, newly installed bike 
lanes did not promote active transportation for residents nearby. The alternative explanation is that one year was too short to observe travel behavior changes (Dill, McNeil, Broach, \& Ma, 2014). Data from three UK municipalities suggested that new bicycle infrastructure was well-used by local adults over a two-year period (Goodman et al., 2013). Bicycle infrastructure planning, mostly adding cycle tracks and separated/buffered bike lanes, partially contributes to the growth of bicycle use by improving individuals' perceptions of safety and comfort (Akar \& Clifton, 2009; Xing, Handy, \& Mokhtarian, 2010). At the micro scale, a wider bike lane is associated with a higher bicycle volume (Fagnant $\&$ Kockelman, 2016). Aside from installing bicycle infrastructure, many complementary interventions, such as car use restrictions, supportive land use planning, and pro-bicycle programs, help promote bicycling. Furthermore, bicycle network design matters to bicycle use, such as the directness of bicycle paths, which is essentially related to trip distance and travel time (Schoner \& Levinson, 2014).

\subsection{Modeling approaches and buffer size selection}

Most existing studies use cross-sectional regression methods to estimate bicycle counts, such as Poisson model, negative binomial model, linear mixed model, binary logit inflation model, ordinary least square regression, and stepwise linear regression (Fagnant \& Kockelman, 2016; Hankey \& Lindsey, 2016; Hankey et al., 2012; Kuzmyak, Walters, Bradley, \& Kockelman, 2014; Niemeier, 1996). In most cases, the trend effect of time-varying variables on bicycle volume change over multiple years is not adequately captured. Hankey and Lindsey (2016) considered the trend effect by aggregating and splitting eight-year bicycle counts into two four-year bicycle counts with one dummy variable. In another study, Lindsey et al. (2008) used months as a categorical variable accounted for the variations in time. To account for spatial autocorrelations, Noland et al. (2016) recently employed a complicated negative binomial conditional autoregressive model to estimate the relationship between bike-sharing usage and the built environment.

Studies using different buffer sizes to quantify built environment features on bicycle counts have failed to reach a consensus. Several studies have considered different buffer sizes to test the consistency of results (Dill \& Voros, 2007; Griswold et al., 2011). Though the buffer sizes vary, the overall trend across studies is that smaller buffers less than one mile, such as 1/10 mile (Griswold et al., 2011), 1/8 mile (Noland, Smart, \& Guo, 2016), 1/4 mile (Dill \& Voros, 2007; Griswold et al., 2011), 1/2 mile (Dill \& Voros, 2007; Griswold et al., 2011), and 1 mile (Dill \& Voros, 2007), are considered to provide more accurate estimation and prediction. Buffer size selection may result in different modeling outcomes. A facility-demand model, which is essentially a modified stepwise linear regression, has been used to allow built environment features quantified at various buffer sizes to be included in the final model (Hankey \& Lindsey, 2016).

In sum, the existing studies have identified a set of determinants on bicycle volume, including bicycle network and other built environment variables, and social demographics. Nevertheless, many of them have not incorporated time-varying factors, largely due to data limitations and modeling constraints. To build upon the previous studies, this paper examines the effects of built environment determinants on the bicycle volume from 2011 to 2015 in the city of Seattle using a GLMM approach.

\section{$3 \quad$ Research design}

\subsection{Study area}

The city of Seattle has already undertaken a considerable effort in promoting bicycling as a more efficient and sustainable transportation mode. The city is continually implementing a bicycle master plan 
which consists of a long-term plan and several short-term implementation plans. By the end of each year, Seattle Department of Transportation (SDOT) produces an annual progress report to demonstrate many performance measures. These measures include (1) ridership; (2) the miles of added bike lanes; (3) safety measured by bicycle collision rate and bicyclist injury severity; (4) connectivity measured by bicycle miles on the road network; (5) equity, measured by accessing areas that lack bicycle facilities; (6) livability, through counting the percentage of biking households in a neighborhood. The measure related to bicycle volume is the annual bike lane increment, which is less than 20 miles per year in Seattle during the past years.

The bicycle count data used in our study were collected from January 2011 to September 2015 by SDOT's program with the goal of better understanding bicycling behavior. The National Bicycle and Pedestrian Documentation methodology was applied and bicycle volume data were collected at 50 selected sites three or four times a year. Designated observers from SDOT were responsible for collecting bicycle counts at intersections. Every four-way intersection had eight designated observers counting bicycles. The data were collected in January, May, July, and September during 10 AM -12 PM, 4 PM -6 PM or 5 PM -7 PM on weekdays and 12 PM -2 PM on Saturdays. Therefore, the bicycle count data are split into three groups: weekday non-peak hours, weekday peak hours, and weekend two hours. In total, each site had bicycles counted 18 times. The July survey was terminated in 2014 . The data were restricted to days without precipitation.

There are several limitations of these data. One potential source of selection bias is the overrepresentation of bicycle-rich locations such as bridges and cycle tracks. A second selection bias is that commuting sites are over-represented in this sample because more locations are observed in downtown Seattle. Therefore, weekend traffic and weekday traffic may not be adequately represented.

To verify the consistency of the relationship between bicycle volume and the built environment, this study considers three buffer radiuses (1-mile, 0.5 -mile, 0.25 -mile) in quantifying built environment factors of each bicycle count site, and compares the consistency of modeling outcomes regarding the changes of corresponding coefficients.

Built environment factors consist of land use and road network characteristics. The sources of the built environment data include SDOT and Puget Sound Regional Council (PSRC). The average bicycle traffic flow over the 18 time periods and the 50sites is displayed in Figures 1 and 2. The count data presented in Figure 2 is the total number of bicycle counts during the 18 time periods.

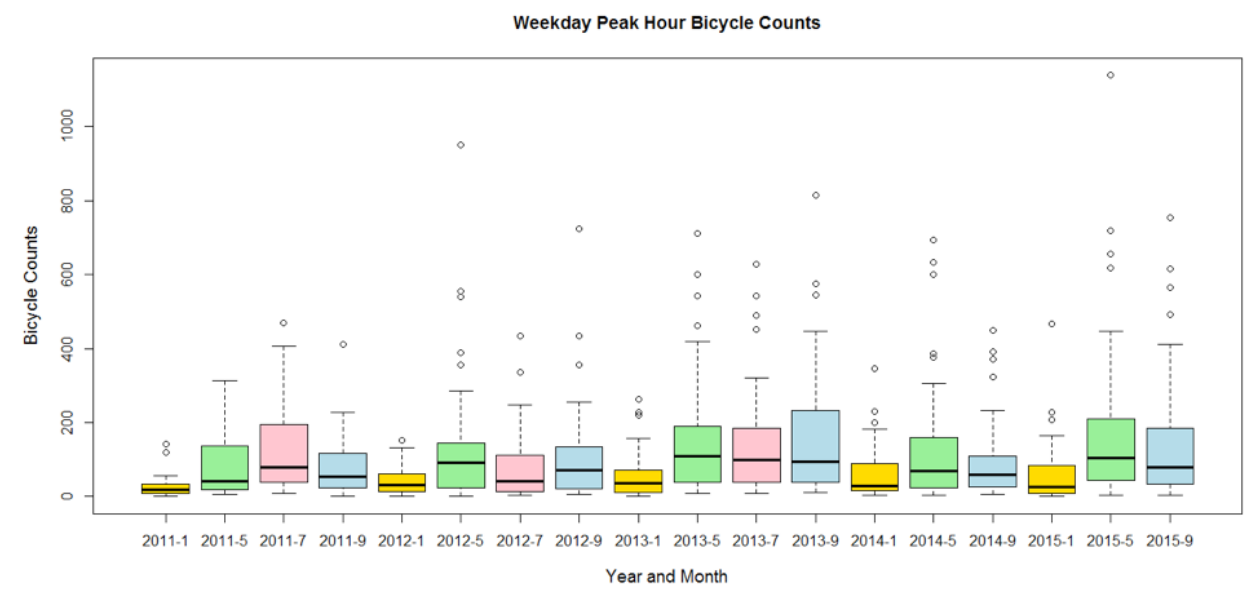

(a) Weekday peak hours (4-6 PM or 5-7 PM) bicycle counts, 2011-2015 


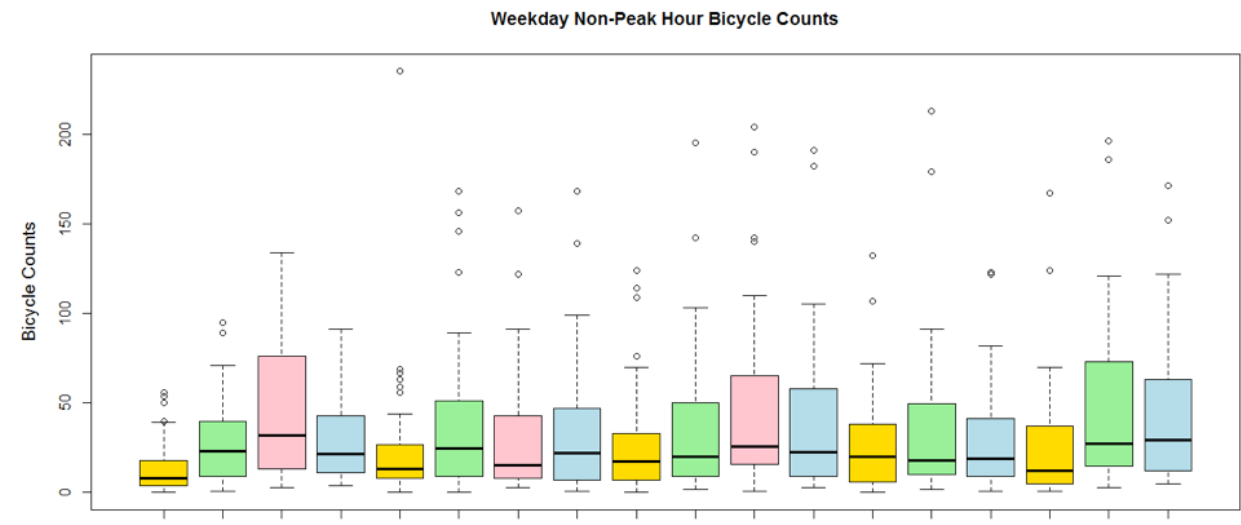

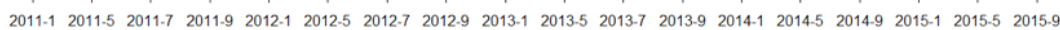
Year and Month

(b) Weekday non-peak hours (10 AM - 12 PM) bicycle counts, 2011-2015

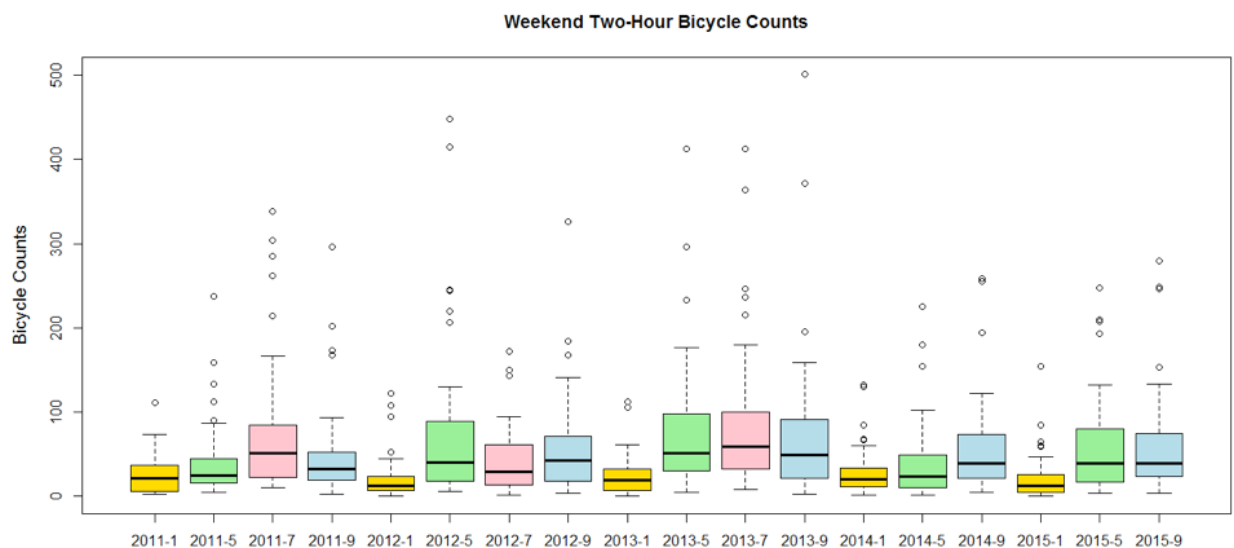

Year and Month

(c) Weekend two hours (12 PM - 2 PM) bicycle counts, 2011-2015

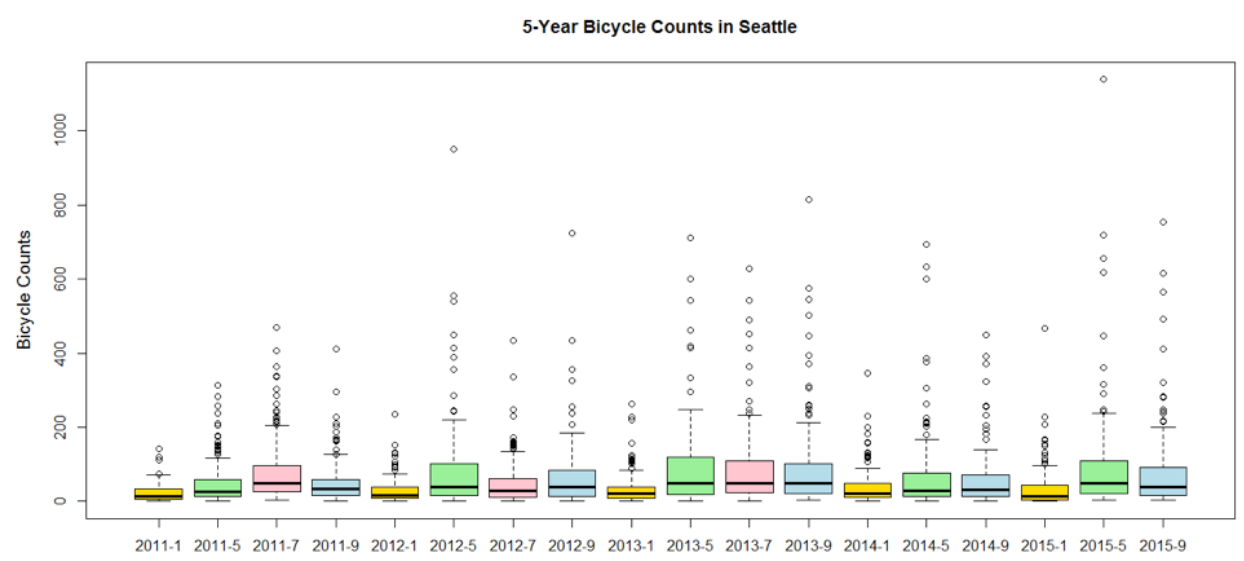
Year and Month

(d) Bicycle counts of all three periods, 2011-2015

Figure 1: Boxplots of Seattle's bicycle counts, 2011-2015 


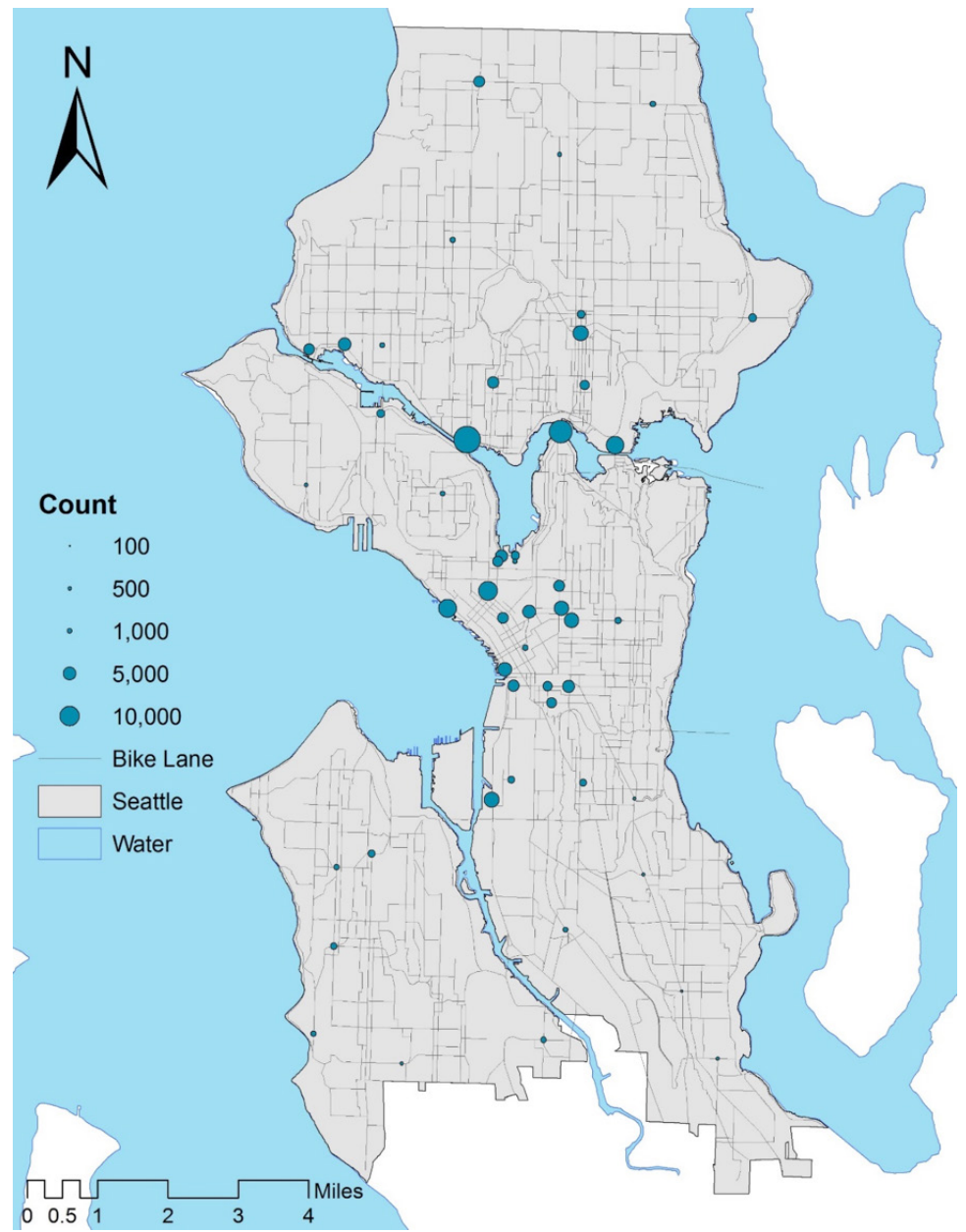

Figure 2: Bicycle count sites and aggregated bicycle counts in Seattle, 2011-2015

\subsection{Longitudinal statistical concerns, GLMM specification, and elasticity calculation}

Bicycle counts change according to hours, days, and seasons. As longitudinal data, the bicycle counts involve the statistical concerns on heteroscedasticity and heterogeneity. To address the above challenges, this study utilizes a generalized linear mixed model (GLMM) approach. GLMM, also called mixed (Poisson) model, is an extension of linear mixed models by allowing response variables to vary for different distributions (Bolker et al., 2009). Thus, it is capable of identifying the temporal autocorrelations and the unobserved heterogeneity through modeling group-specific variability in trajectories over time and subject-specific differences (Qureshi \& Fang, 2010). Since GLMM is not commonly adopted in bicycle use studies, to better illustrate its strengths, the statistical concerns and the corresponding modeling strategies are discussed in detail as follows.

First, when bicycle counts are repeatedly measured at the same site over time, the values are likely to be highly correlated, and the estimated curve is expected to deviate systematically from the true trend curve, resulting in the heteroscedasticity, which violates the independence assumption of general fixedeffects models. GLMM resolves the issue of heteroscedasticity through interpreting the interaction effects of time metric and time-varying variables. 
Second, heterogeneity signifies the diversity of subjects. Regarding the data, while a fixed-effect model can capture the observed variations across different bicycle count sites, it fails to account for the unobserved heterogeneity of each subject. In contrast, the inclusion of random effects in this GLMM can account for the unobserved heterogeneity.

Another benefit of including random effects in the GLMM is to resolve the problem of data overdispersion. Because bicycle count is a count data, the distribution of response variables (especially across sites) is assumed to follow a Poisson distribution. As an issue of count data, accounting for over-dispersion is vital to the accuracy of the modeling outcome.

In this model, the time metric is defined by "the month divided by 12 plus the year and minus 2011," ranging from 0.08 to 4.75 years. In total, each bicycle count site has 18 measurement occasions from January 2011 to September 2015 with a generally fixed schedule.

This GLMM helps estimate the joint effect of the built environment, the natural environment, socio-demographics, and time-varying variables on bicycle counts, expressed by Eq. (1) and Figure 3 (Mustillo et al., 2012). Thus, a conditional expectation function model can predict changes in log bicycle counts at site $i$ and occasion $t$. This GLMM has subject-specific intercepts and slopes, which allows each bicycle count site to vary by the initial level of bicycle volume and the rate of change over time. Eq. (2) represents the level-1 model, which follows the traditional linear model specification by assuming the logarithmic link function $\ln \left(\mu_{i t}\right)$. The level-2 model estimates the fixed effects and the random effects that depart from group-specific changes with subject-specific intercepts and slopes that are estimated from the level-1 model, as shown in Eqs. (3) and (4). The composite model is specified as Eq. (5).

$$
\begin{aligned}
& \mathrm{E}\left(\mathrm{Y}_{\mathrm{it}}\right)=\mu_{\mathrm{it}}=\exp \left(\beta_{0 \mathrm{i}}+\beta_{\mathrm{li}} \text { Time }_{\mathrm{it}}\right) \\
& \ln \left(\mu_{\mathrm{it}}\right)=\beta_{0 \mathrm{i}}+\beta_{1 \mathrm{i}} \text { Time }_{\mathrm{it}} \\
& \beta_{0 \mathrm{i}}=\beta_{00}+\beta_{0 i} \mathrm{X}_{\mathrm{it}}+\zeta_{0 \mathrm{i}} \\
& \beta_{1 \mathrm{i}}=\beta_{10}+\beta_{1 i} \mathrm{X}_{\mathrm{it}}+\zeta_{1 \mathrm{i}} \\
& \ln \left(\mu_{\mathrm{it}}\right)=\beta_{00}+\beta_{0 i} \mathrm{X}_{\mathrm{it}}+\beta_{10} \text { Time }_{\mathrm{it}}+\beta_{1 i} \mathrm{X}_{\mathrm{it}} \text { Time }_{\mathrm{it}}+\zeta_{0 \mathrm{i}}+\zeta_{\mathrm{li}} \text { Time }_{\mathrm{it}}
\end{aligned}
$$

Where $\beta_{o i}$ and $\beta_{I i}$ are the Poisson estimates that vary across bicycle count sites $i ; \mu_{i t}$ is the mean of bicycle counts at the 50 selected sites over the 18 occasions $t ; \beta_{o 0}$ is the overall intercept; Time $e_{i t}$ is a level-1 time variable, with the fixed effects parameters $\beta_{1 i} X_{i}$ refers to the fixed effects, specifically referring to the time-varying variables, socio-demographic factors, natural environment features, and built environment features; $X_{i}^{*}$ Time $_{i t}$ is the cross-level product for time changes that interact with the fixed effects coefficients $\beta_{1 i} \zeta_{O i}$ and $\zeta_{1 i}$ are random effects of each subject that depart from group-averaged intercept and slope, respectively. Only time-varying variables are included for modeling the cross-level interaction effects. In addition, random effects are assumed to follow a multivariate normal distribution.

To measure the factor proportional substitution effects, elasticities are computed for the fixed effects. The direct-elasticity of continuous variables for a Poisson model is computed using Eq. (6) (Ewing \& Cervero, 2010):

$$
E_{\text {Direct }}=\beta \overline{\mathrm{X}}
$$




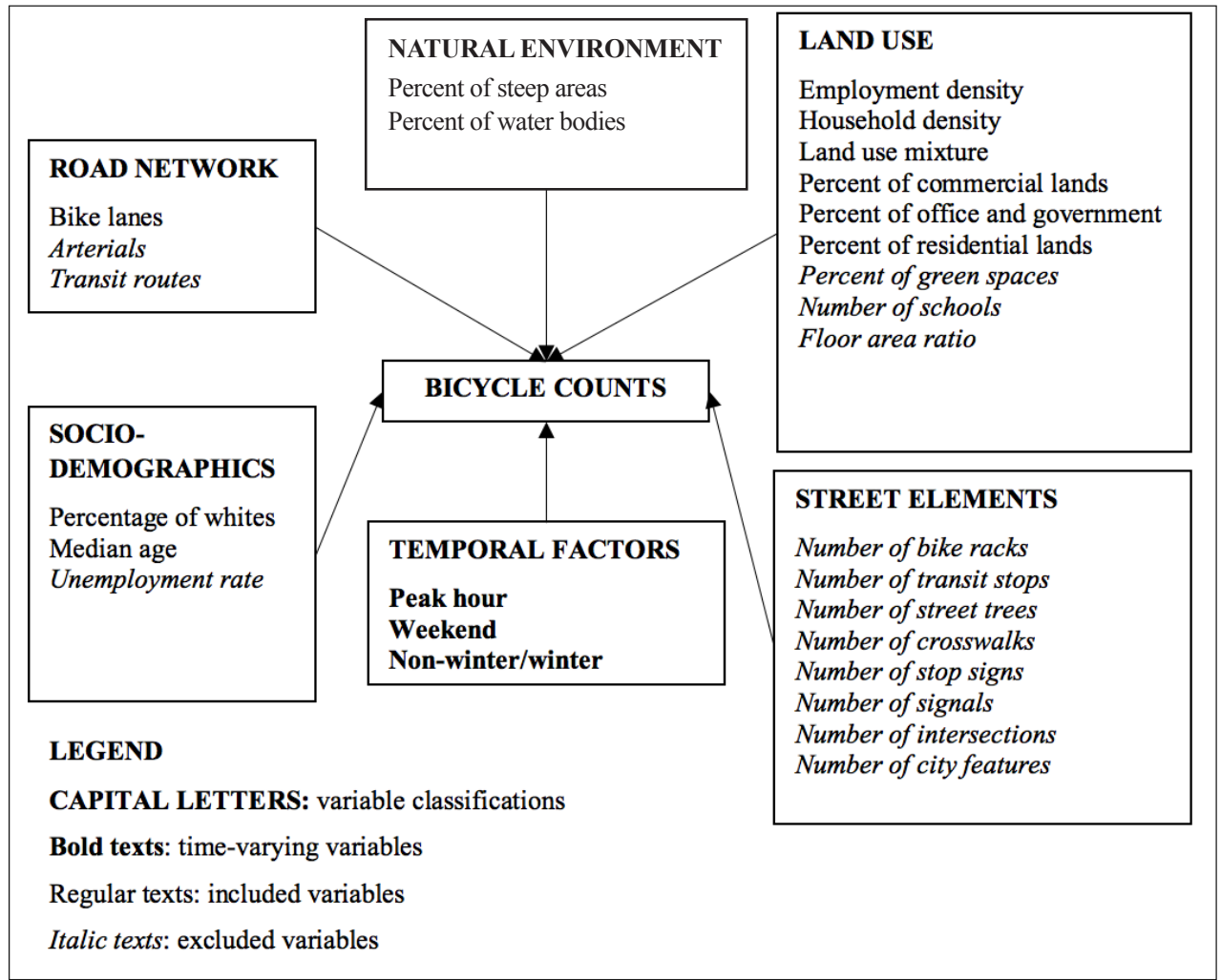

Figure 3: Modeling framework

\subsection{Variable selection}

Three time-varying variables are considered for interaction effects in the final model, including nonwinter/winter seasons, peak hours, and weekends. This study initially considers a large set of fixed covariates, but many variables are excluded due to multicollinearities. The variance inflation factor (VIF) is used to check for multicollinearity, and variables with VIF values greater than 5.0 are excluded (Brooks \& Barcikowski, 1999)

Variable selection is based on two criteria. First, more important explanatory variables related to land use and road networks, such as the length of bike lanes and land use mixture, are given priorities in selection. For example, floor area ratio (FAR) and employment density show high multi-collinearity in modeling, and dropping one of them decreases the VIF value of another. Employment density is a more commonly used index in transportation planning research in the US, though FAR is measured at the site level with parcel data and expected to be more accurate. To facilitate the comparison with other studies, this study drops FAR but keeps employment density in the final model. For the built environment, road network factors excluded are the cumulative length of arterial routes and the cumulative length of transit routes, which are highly correlated with the cumulative length of bike lanes. Second, variables with greater VIF values are prioritized in exclusion. Street elements excluded are the number of bus stops, stop signs, road intersections, bike racks, traffic signals, city features, and street trees. Education rate and unemployment rate are excluded for their high correlations with racial composition. 


\section{$4 \quad$ Results}

\subsection{Descriptive analysis}

The definitions of variables and data summary are listed in Tables 1, 2, and 3. SDOT counted bicycles from January 2011 to September 2015. SDOT gathered 70\% of the bicycle counts from May to September; $33 \%$ of the data from 4 PM -6 PM or 5 PM -7 PM to reflect peak hour variation; and 33\% of the data on Saturdays to differentiate weekdays and weekends.

The two-hour bicycle count data summary is presented in Table 2. As noted, more bicycle counts are observed during weekday peak hours, followed by weekends, and then weekday non-peak hours. To clarify, the average counts in weekday peak hours (4-6 PM or 5-7 PM) are 100. 48 bicycles, the average counts in weekend two hours (12 PM - 2 PM) are 50.71 bicycles, and the average counts in weekday non-peak hours (10 AM - 12 PM) are 33.60 bicycles. Bicycle counts, as expected, reach its peak in July and its trough in January.

The other included variables are measured with different buffer radiuses. The percentage of whites, the median age, the percentage of residential lands, and household density are mostly constant across the three spatial scales regardless of buffer radius. The percentage of commercial lands, the percentage of offices, and employment density increase as the buffer radius decreases, while the percentage of water bodies, the percentage of steep area, and land use mixture decrease as the buffer radius decreases.

Table 1: Variable description

\begin{tabular}{|l|l|l|}
\hline & \multicolumn{1}{|c|}{ Source } & \multicolumn{1}{|c|}{ Description } \\
\hline Bicycle counts & SDOT & The number of bicycle counts \\
\hline Year \& Month - 2011 & SDOT & The month divided by 12 plus the year minus 2011; \\
\hline Non-winter/Winter & SDOT & If bicycles are not counted in winter, 1, else 0; \\
\hline Peak hour & SDOT & If bicycles counted in 4-6 PM or 5-7 PM, 1, else 0; \\
\hline Weekend & SDOT & If bicycles counted on Saturday, 1, else 0; \\
\hline Bike lane & SDOT & The sum of bike lane length, in miles; \\
\hline Pct. of steep areas & SDOT & $\begin{array}{l}\text { The percent of steep areas in buffers, in \%; Seattle's Design \& Planning Depart- } \\
\text { ment designates steep areas, and slopes greater than or equal to 40 percent are } \\
\text { counted as steep areas. }\end{array}$ \\
\hline Median age & ACS & The median age in neighborhoods; \\
\hline Pct. of white & ACS & The percent of whites, in \%; \\
\hline Pct. of water & PSRC & The percent of water spaces in buffers, in \%; \\
\hline Pct. of commercial & PSRC & The percent of commercial and mixed land use in buffers, in \%; \\
\hline Pct. of residential & PSRC & The percent of residential lands in buffers, in \%; \\
\hline LUM & PSRC & $\begin{array}{l}\text { The entropy of six types of land use, including residential, green spaces, commer- } \\
\text { cial, offices, and water bodies, within buffers of bicycle count sites, in \%; }\end{array}$ \\
\hline Pct. of green & PSRC & The percent of green spaces and parks in buffers, in \%; \\
\hline Pct. of office & PSRC & The percent of offices and governments in buffers, in \%; \\
\hline Employ density & PSRC & The number of employments per square mile; \\
\hline Household density & PSRC & The number of households per square mile. \\
\hline $\begin{array}{l}\text { SDOT: Seattle Department of Transportation } \\
\text { PSRC: Puget Sound Regional Council } \\
\text { ACS: American Community Survey }\end{array}$ & \\
\hline
\end{tabular}


Table 2: Descriptive analysis of bike counts in different time periods

\begin{tabular}{|l|c|c|c|c|}
\hline Bike counts & Mean & S.D. & Min. & Max. \\
\hline All three periods & 61.59 & 90.22 & 0.00 & 1138.00 \\
\hline Weekday non-peak hours & 33.60 & 35.93 & 0.00 & 235.00 \\
\hline Weekday peak hours & 100.48 & 130.02 & 0.00 & 1138.00 \\
\hline Weekend two hours & 50.71 & 61.73 & 0.00 & 501.00 \\
\hline Winter season & 32.11 & 42.57 & 0.00 & 467.00 \\
\hline Non-winter seasons & 72.88 & 100.52 & 0.00 & 1138.00 \\
\hline
\end{tabular}

Table 3: Data summary for selected variables

\begin{tabular}{|c|c|c|c|c|c|c|c|c|c|c|c|c|}
\hline & \multicolumn{4}{|c|}{ 1-mile buffer } & \multicolumn{4}{|c|}{0.5 -mile buffer } & \multicolumn{3}{c|}{0.25 -mile buffer } \\
\cline { 2 - 14 } & Mean & S.D. & Min. & Max. & Mean & S.D. & Min. & Max. & Mean & S.D. & Min. & Max. \\
\hline Year \& Month - 2011 & 2.28 & 1.39 & 0.08 & 4.75 & 2.28 & 1.39 & 0.08 & 4.75 & 2.28 & 1.39 & 0.08 & 4.75 \\
\hline Non-winter/ Winter & 0.72 & 0.45 & 0.00 & 1.00 & 0.72 & 0.45 & 0.00 & 1.00 & 0.72 & 0.45 & 0.00 & 1.00 \\
\hline Peak Hour & 0.33 & 0.47 & 0.00 & 1.00 & 0.33 & 0.47 & 0.00 & 1.00 & 0.33 & 0.47 & 0.00 & 1.00 \\
\hline Weekend & 0.33 & 0.47 & 0.00 & 1.00 & 0.33 & 0.47 & 0.00 & 1.00 & 0.33 & 0.47 & 0.00 & 1.00 \\
\hline Bike lane & 22.14 & 5.76 & 7.44 & 31.14 & 6.23 & 1.99 & 2.48 & 10.20 & 1.76 & 0.63 & 0.52 & 3.63 \\
\hline Pct. of steep areas & 5.35 & 3.14 & 1.26 & 12.97 & 3.56 & 3.03 & 0.00 & 11.33 & 2.61 & 2.94 & 0.00 & 14.12 \\
\hline Median age & 36.34 & 3.59 & 23.32 & 42.14 & 36.59 & 4.83 & 23.39 & 47.56 & 36.25 & 5.40 & 23.21 & 47.94 \\
\hline Pct. of whites & 67.12 & 15.62 & 25.46 & 88.20 & 66.25 & 18.39 & 13.76 & 89.29 & 64.65 & 20.39 & 12.84 & 89.40 \\
\hline Pct. of water bodies & 10.99 & 10.66 & 0.00 & 48.80 & 7.71 & 11.73 & 0.00 & 46.30 & 5.85 & 10.89 & 0.00 & 47.94 \\
\hline Pct. of commercial & 6.64 & 5.41 & 0.20 & 19.19 & 8.98 & 9.24 & 0.09 & 34.24 & 13.05 & 13.51 & 0.00 & 53.87 \\
\hline Pct. of residential & 13.19 & 7.86 & 2.98 & 36.09 & 13.07 & 11.62 & 1.43 & 44.70 & 13.23 & 13.21 & 0.80 & 49.54 \\
\hline LUM & 66.42 & 12.23 & 42.30 & 86.55 & 61.88 & 13.44 & 39.01 & 86.22 & 60.65 & 11.82 & 38.22 & 82.93 \\
\hline Pct. of offices & 7.76 & 5.43 & 0.48 & 19.97 & 9.77 & 10.71 & 0.31 & 49.06 & 10.19 & 13.49 & 0.00 & 65.30 \\
\hline Employment density & 27.24 & 29.09 & 1.17 & 97.80 & 30.05 & 40.10 & 1.06 & 175.64 & 33.92 & 49.77 & 1.68 & 245.58 \\
\hline Household density & 8.92 & 5.93 & 0.09 & 20.73 & 9.48 & 6.95 & 0.00 & 27.82 & 9.31 & 7.26 & 0.00 & 32.02 \\
\hline
\end{tabular}




\subsection{Inferential analysis}

Table 4: GLMM modeling outcomes (the number of observations=2,687, the number of groups=50), and calculated elasticities

\begin{tabular}{|c|c|c|c|c|c|c|c|c|c|}
\hline & \multicolumn{3}{|c|}{ 1-mile buffer } & \multicolumn{3}{|c|}{ 0.5-mile buffer } & \multicolumn{3}{|c|}{ 0.25-mile buffer } \\
\hline & Estimate & $\mathrm{P}$-value & Elasticity & Estimate & P-value & Elasticity & Estimate & $\mathrm{P}$-value & Elasticity \\
\hline (Intercept) & 0.47 & 0.75 & & 1.11 & 0.34 & & $2.24^{*}$ & 0.01 & \\
\hline Year \& Month -2011 & $0.09^{* * *}$ & 0.00 & & $0.09^{* * *}$ & 0.00 & & $0.09^{* * *}$ & 0.00 & \\
\hline Non-winter/Winter & $0.90^{* * *}$ & 0.00 & & $0.90^{* * *}$ & 0.00 & & $0.90^{* * *}$ & 0.00 & \\
\hline Peak hour & $0.94^{* * *}$ & 0.00 & & $0.94^{* * *}$ & 0.00 & & $0.94^{* * *}$ & 0.00 & \\
\hline Weekend & $0.49^{* * *}$ & 0.00 & & $0.49^{* * *}$ & 0.00 & & $0.49^{* * *}$ & 0.00 & \\
\hline $\begin{array}{l}\text { Year \& Month - 2011: } \\
\text { Season }\end{array}$ & $-0.04^{* * *}$ & 0.00 & & $-0.04^{* * *}$ & 0.00 & & $-0.04^{* * *}$ & 0.00 & \\
\hline $\begin{array}{l}\text { Year \& Month - 2011: Peak } \\
\text { Hour }\end{array}$ & $0.06^{* * *}$ & 0.00 & & $0.06^{* * *}$ & 0.00 & & $0.06^{* * *}$ & 0.00 & \\
\hline $\begin{array}{l}\text { Year \& Month - 2011: } \\
\text { Weekend }\end{array}$ & $-0.04^{* * *}$ & 0.00 & & $-0.04^{* * *}$ & 0.00 & & $-0.04^{* * *}$ & 0.00 & \\
\hline Bike lane & $0.04^{*}$ & 0.05 & 0.88 & 0.09 & 0.12 & 0.56 & $0.34^{*}$ & 0.03 & 0.59 \\
\hline Pct. of steep areas & $-0.13^{* *}$ & 0.00 & -0.71 & -0.01 & 0.86 & -0.02 & -0.02 & 0.58 & -0.05 \\
\hline Median age & -0.06 & 0.07 & -2.22 & $-0.05^{*}$ & 0.03 & -1.88 & $-0.05^{* *}$ & 0.00 & -1.96 \\
\hline Pct. of whites & $0.03^{* * *}$ & 0.00 & 1.77 & $0.02^{*}$ & 0.01 & 1.03 & $0.01^{*}$ & 0.05 & 0.66 \\
\hline Pct. of water bodies & $0.03^{*}$ & 0.03 & 0.32 & $0.03^{*}$ & 0.02 & 0.22 & $0.05^{* * *}$ & 0.00 & 0.27 \\
\hline Pct. of commercial & -0.03 & 0.25 & -0.22 & -0.01 & 0.48 & -0.12 & 0.00 & 0.89 & -0.02 \\
\hline Pct. of residential & 0.03 & 0.15 & 0.34 & 0.01 & 0.60 & 0.08 & 0.00 & 0.73 & 0.04 \\
\hline LUM & $0.02 *$ & 0.03 & 1.61 & 0.01 & 0.30 & 0.76 & 0.00 & 0.91 & 0.07 \\
\hline Pct. of offices & & & & 0.02 & 0.17 & 0.19 & $0.02^{*}$ & 0.01 & 0.24 \\
\hline Employment density & & & & 0.00 & 0.96 & 0.01 & 0.00 & 0.75 & 0.02 \\
\hline Household density & & & & 0.03 & 0.10 & 0.32 & 0.02 & 0.31 & 0.15 \\
\hline \multicolumn{10}{|c|}{ Level of significance: “***”, $0.1 \%$; “**”, 1\%; “*”, “5\%”. } \\
\hline Random effects & \multicolumn{9}{|c|}{ Location ID } \\
\hline Model fit & Variance & AIC & $\mathrm{BIC}$ & Variance & AIC & $\mathrm{BIC}$ & Variance & AIC & $\mathrm{BIC}$ \\
\hline & 0.3857 & $50,570.4$ & $50,670.7$ & 0.3639 & $50,537.5$ & $50,691.4$ & 0.327 & $50,565.7$ & $50,689.5$ \\
\hline & Log Link & Deviance & DF. residual & Log Link & Deviance & DF. residual & Log Link & Deviance & DF. residual \\
\hline & $-25,268.2$ & $50,536.4$ & $2,670.0$ & -25266.8 & $50,533.5$ & $2,667.0$ & $-25,261.8$ & $50,523.7$ & $2,666.0$ \\
\hline
\end{tabular}

As shown in Table 4, the outcome indicates that two natural environment factors and two built environment factors - the percent of steep areas, the percent of water bodies, land use mixture, and bike lane length - are significantly correlated with bicycle counts. The percent of steep areas is negatively associated with bicycle counts. Hilly areas discourage bicycling, but such an effect is only significant when the percent of steep areas is measured at the 1-mile buffer. Bike lane length and land use mixture are positive predictors of bicycle counts. Specifically, increasing land use mixture by $1 \%$ is associated with a $1.61 \%$ higher bicycle counts, such an effect is only significant for the 1-mile buffer. Compared with the above, the effect of bike lane length in promoting bicycle volume is more consistent across the three buffer radiuses. A higher percentage of mixed land use creates opportunities to load more bicycle traffic. In areas closer to water bodies, including seas, lakes, rivers, and creeks, bicycle counts are greater. The effects of commercial and residential lands on bicycle counts are not significant.

Socio-demographic factors are important predictors of bicycle counts. Bicycling appears to be more popular in neighborhoods with higher percentages of white and younger people. A $1 \%$ increase in the percentage of white associates with an increase of bicycle counts ranging from $0.66 \%$ to $1.77 \%$, as the built environment is measured from 1-mile, 0.5 -mile to 0.25 -mile buffers. Similarly, a $1 \%$ increase in 
the median age associates with a decrease of bicycle counts ranging from $1.88 \%$ to $2.22 \%$.

The interpretations of the time-varying variables are based on the interaction effects of the time metrics and the time-varying variables, while fixing the other effects to be constant in predictions, as shown in Figure 4. Since the coefficients for interaction terms are equal, this study only presents the prediction based on coefficients modeled from the 1-mile buffer data. The intercept of a time-varying variable indicates the initial difference from the trend curve. The coefficient estimated for the interaction term is the expected rate of change, which is measured by the deviate of conditional expectation of an interaction term with respect to the time metric.

As indicated in the three prediction curves, bicycle counts have been steadily increasing over the research period conditional on the effects of season, peak hours, and weekends. For the seasonal effect, as shown in Table 4 and Figure 4 (a), bicycle counts are increasing at a slightly lower rate but a much greater volume over time in non-winter seasons. The initial bicycle counts are greater in peak hours, and the changing rate of bicycle counts is much higher in peak hours over time, as shown in Table 4 and Figure 4 (b). When the other covariates are fixed, the predicted bicycle counts are greater on weekends, but the increasing rate is slightly lower than that during weekdays, as shown in Table 4 and Figure 4 (c).

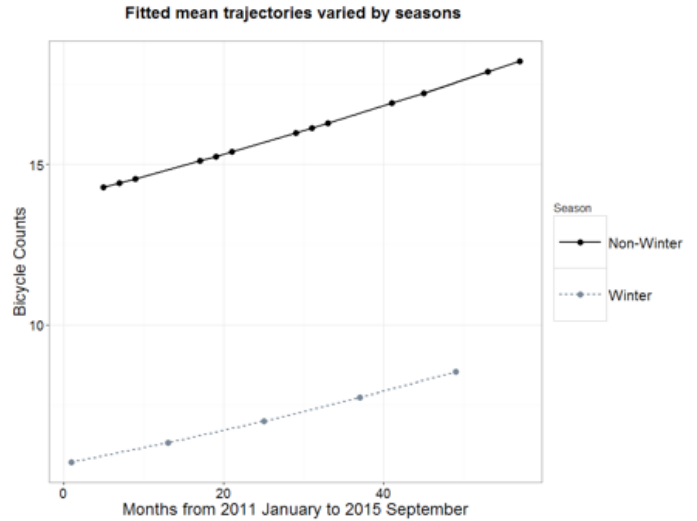

(a) Non-winter/Winter

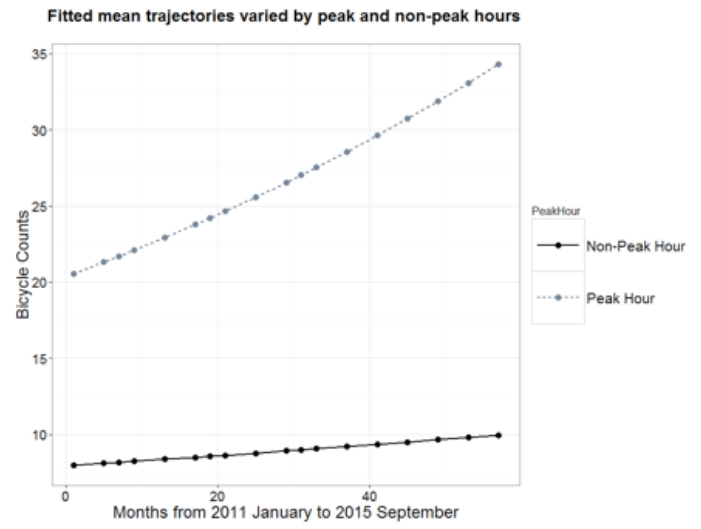

(b) Weekday 4-6 or 5-7 PM (peak hours)

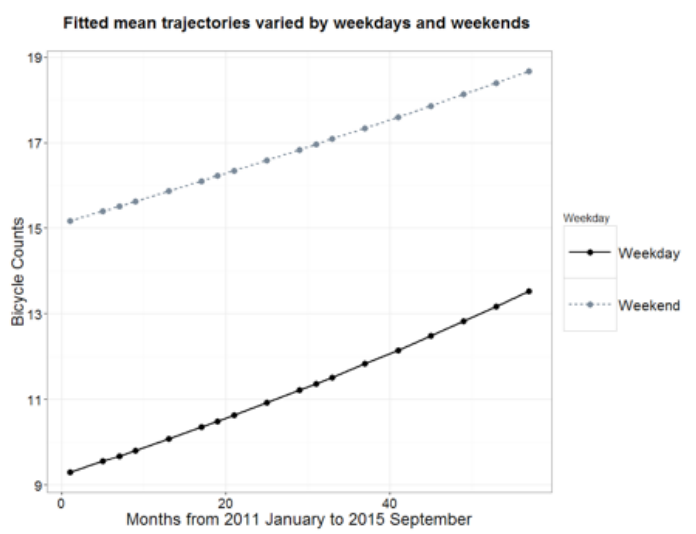

(c) Weekday/Weekend

Figure 4: The fitted mean trajectories of the time metric interacted with three time-varying variables

As for random effects, the overall intercept is considered to explain the subject-specific variations. The equation to calculate the intra-class correlation (ICC) for a GLMM is expressed by Eq. (7) (Stryhn, Sanchez, Morley, Booker, \& Dohoo, 2006). 


$$
\mathrm{ICC}=\frac{\left[\exp \left(2 \mathrm{X}_{i} \beta_{i}+2 \sigma^{2}\right)-\exp \left(2 \mathrm{X}_{i} \beta_{i}+\sigma^{2}\right)\right]}{\left[\exp \left(2 \mathrm{X}_{i} \beta_{i}+2 \sigma^{2}\right)-\exp \left(2 \mathrm{X}_{i} \beta_{i}+\sigma^{2}\right)+\exp \left(\mathrm{X}_{i} \beta_{i}+\sigma^{2} / 2\right)\right]}
$$

Where $\sigma^{2}$ is the total variance of the residual and the random effects.

The calculated ICCs for the three models are $92.47 \%$ (1-mile buffer), $85.68 \%$ (0.5-mile buffer), and $62.68 \%(0.25$-mile buffer). The ICCs are all greater than $50 \%$, indicating that the total variation is attributable to site-specific differences. The higher the ICC, the greater association it is between any pair of bicycle counts on the same location. It confirms the assumption that temporal autocorrelations exist among multiple bicycle counts from the same location.

\section{$5 \quad$ Conclusions and discussion}

This study uses Seattle's longitudinal bicycle count data to examine the effect of built environment factors on bicycle volume change over time. The results show that time-varying variables-non-winter seasons, peak hours, and weekends - are essential determinants of bicycle traffic. In addition, this study identifies and addresses significant temporal autocorrelations of bicycle counts within sites over five years, which indicates that the longitudinal correlations must be adjusted to avoid possible biased estimations when modeling the relationship between bicycle counts and the other fixed covariates.

For planning practices, the coefficient estimated for the length of bike lanes is significant, which partially confirms a popular opinion on bicycle infrastructures: "if you build them, people will use them" (Dill \& Carr, 2003). This also provides some evidence to show the benefit of continually implementing a bicycle master plan. For land use mixture and mountainous terrains, the results of this study are consistent with previous findings. Areas with a more mixed land use (Hankey et al., 2012) and a higher percentage of water bodies are positively associated with greater bicycle counts, while a hilly terrain is negatively correlated with bicycle counts (Griswold et al., 2011). These findings inform where cities should give priority to densifying bike infrastructures. In addition, newly added bike lanes may be placed in areas with a more mixed land use or areas with a greater access to water bodies. Cities could either prioritize building bike lanes in flat areas because they are more likely to be used, or at least add specifically-treated climbing lanes which better secure bicyclists from collisions when they are moving up hills.

The incorporation of time-varying variables provides interesting results. The overall result shows a growing trend of bicycle counts in Seattle. It is encouraging to see that such a trend not only occurs in non-winter seasons, peak-hours, or weekends, but also appears in winter, non-peak hours, and workdays when people are less likely to bike. The climbing curves indicate an increasing popularity of bicycling in culture and the effectiveness of the government's efforts in enhancing the bicycling environment. The result is expected to provide policies and applications to build and manage infrastructures for bicycling in different time occasions.

Neighborhood racial and age compositions are correlated with bicycle use. Despite a higher number of bikers observed in neighborhoods with more young and white people, we still do not know who these bicyclists are or in which neighborhoods they are living. To better understand the demographic of bicyclists, a more insightful survey is needed to complement the investigation of this study. With the equity concern, authorities also need to provide more supporting infrastructure in underserved communities. Last, but not least, we need to further study why the bicycle use varies across races and ages and what we can do to promote more bicycle use among the disadvantaged neighborhoods and social groups.

Another important limitation to be noted is the spatial and temporal biases of data collection. The data is collected in different weekdays or weekends in different seasons. So far, no reasonable weight can be assigned to account for the time effect in aggregating bicycle counts in models. It is hard to argue 
between weekday peak hours, weekday non-peak hours, and weekend two-hours bicycle counts, which period is more important for estimation and prediction. In addition, location selection is another source of sample bias. These problems should be addressed by strategic data collection.

Future studies can be improved in three aspects. First, more longitudinal data should be collected to understand the temporal effects. Five-years is still a relatively short period for a longitudinal analysis of the effect of the built environment on bicycle usage. Most built environment features could remain unchanged in five years in a developed country like the US. Though bike lanes have been densified in Seattle, such changes may not result in dramatic differences in bicycle-count trajectories, and therefore the accumulated changes of bicycle count over a long time should not be underestimated. Second, better-measured built environment variables could be included. Some built-environment factors are excluded due to multicollinearity in this analysis, such as road intersection density and floor area ratio. Studies could develop some composite measurements for those excluded variables. Of course, the interpretation of composite measures for policy implications is always challenging, but it provides an alternative way to gain more insights from the data. Third, future studies may consider a more advanced model to control spatial autocorrelations. The GLMM is powerful in capturing temporal effects, but it cannot account for spatial autocorrelations. The bicycle count data may suffer from observation sites clustered in urban centers. Geographically-approximated bicycle count sites, or different locations on a bike trail, may report similar numbers of bicycle counts, which violate the assumption that the fixed effects should be independent and identically distributed (Gibbons, Hedeker, \& DuToit, 2010). A more advanced data collection and modeling approach could better address these statistical concerns.

Note

LUM: the degree of mixing land use is measured by

$$
L U M=-\sum P_{i} * \ln P_{i} / \ln n
$$

Where $n$ is the number of different land use types and $P_{i}$ is the proportion of land in type $i$. This index is calculated separately for each zone. LUM ranges from $O$ (homogeneous land use) to $I$ (most mixed land use). 


\section{References}

Akar, G., \& Clifton, K. (2009). Influence of individual perceptions and bicycle infrastructure on decision to bike. Transportation Research Record, 2140, 165-172.

Bachand-Marleau, J., Larsen, J., \& El-Geneidy, A. (2011). Much-anticipated marriage of cycling and transit: How will it work? Transportation Research Record, 2247, 109-117.

Bhatia, R., \& Wier, M. (2011). "Safety in numbers" re-examined: Can we make valid or practical inferences from available evidence? Accident Analysis \& Prevention, 43(1), 235-240.

Bolker, B. M., Brooks, M. E, Clark, C. J, Geange, S. W, Poulsen, J. R., Stevens, M H., \& White, J. S. (2009). Generalized linear mixed models: A practical guide for ecology and evolution. Trends in ecology \& evolution, 24(3), 127-135.

Brooks, G. P., \& Barcikowski, R. S. (1999). The precision efficacy analysis for regression sample size method. Paper presented at the meeting of the American Educational Research Association, Montreal, Quebec, Canada.

Buehler, R. (2012). Determinants of bicycle commuting in the Washington, DC region: The role of bicycle parking, cyclist showers, and free car parking at work. Transportation research part D: Transport and Environment, 17(7), 525-531.

Buehler, R., \& Pucher, J. (2012). Cycling to work in 90 large American cities: New evidence on the role of bike paths and lanes. Transportation, 39(2), 409-432.

Buehler, R., Pucher, J., Merom, D., \& Bauman, A. (2011). Active travel in Germany and the U.S.: Contributions of daily walking and cycling to physical activity. American Journal of Preventive Medicine, 41(3), 241-250. doi: http://dx.doi.org/10.1016/j.amepre.2011.04.012

Chen, P. (2015). Built environment factors in explaining the automobile-involved bicycle crash frequencies: A spatial statistic approach. Safety Science, 79, 336-343. doi: http://dx.doi.org/10.1016/j. ssci.2015.06.016

Chen, P., \& Shen, Q. (2016). Built environment effects on cyclist injury severity in automobile-involved bicycle crashes. Accident Analysis \& Prevention, 86, 239-246. doi: http://dx.doi.org/10.1016/j. aap.2015.11.002

dell'Olio, L., Ibeas, A., Bordagaray, M., \& Ortúzar, J. de D. (2013). Modeling the effects of pro bicycle infrastructure and policies toward sustainable urban mobility. Journal of Urban Planning and Development, $140(2), 04014001$.

Dill, J., \& Carr, T. (2003). Bicycle commuting and facilities in major U.S. cities: If you build them, commuters will use them. Transportation Research Record, 1828(-1), 116-123. doi: 10.3141/1828-14

Dill, J., McNeil, N., Broach, J., \& Ma, L. (2014). Bicycle boulevards and changes in physical activity and active transportation: Findings from a natural experiment. Preventive medicine, 69, S74-S78.

Dill, J., \& Voros, K. (2007). Factors affecting bicycling demand: Initial survey findings from the Portland, Oregon, region. Transportation Research Record, 2031(-1), 9-17. doi: 10.3141/2031-02

El Esawey, M., Lim, C., Sayed, T., \& Mosa, A. I. (2013). Development of daily adjustment factors for bicycle traffic. Journal of Transportation Engineering, 139(8), 859-871.

El Esawey, M., Mosa, A. I., \& Nasr, K. (2015). Estimation of daily bicycle traffic volumes using sparse data. Computers, Environment and Urban Systems, 54, 195-203.

Ewing, R., \& Cervero, R.. (2010). Travel and the built environment: A meta-analysis. Journal of the American Planning Association, 76(3), 265-294.

Faghih-Imani, A., Eluru, N., El-Geneidy, A. M., Rabbat, M., \& Haq, U. (2014). How land-use and urban form impact bicycle flows: evidence from the bicycle-sharing system (BIXI) in Montreal. Journal of Transport Geography, 41, 306-314. 
Fagnant, D. J., \& Kockelman, K. (2016). A direct-demand model for bicycle counts: The impacts of level of service and other factors. Environment and Planning B: Planning and Design, 43(1). 93-107.

Gallop, C., Tse, C,, \& Zhao, J. (2012). A seasonal autoregressive model of Vancouver bicycle traffic using weather variables. Paper presented at the Transportation Research Board 91st Annual Meeting. Washington, DC.

Gibbons, R. D., Hedeker, D., \& DuToit, S. (2010). Advances in analysis of longitudinal data. Annual Review of Clinical Psychology, 6, 79-107. doi: 10.1146/annurev.clinpsy.032408.153550

Goetzke, F., \& Rave, T. (2010). Bicycle use in Germany: Explaining differences between municipalities with social network effects. Urban Studies, 48(2), 427-437.

Goodman, A., Sahlqvist, S., Ogilvie, D., \& consortium, iConnect. (2013). Who uses new walking and cycling infrastructure and how? Longitudinal results from the UK iConnect study. Preventive Medicine, 57(5), 518-524.

Gosse, C, \& Clarens, A. (2014). Estimating spatially and temporally continuous bicycle volumes by using sparse data. Transportation Research Record, 2443, 115-122.

Griswold, J., Medury, A., \& Schneider, R. (2011). Pilot models for estimating bicycle intersection volumes. Transportation Research Record, 2247, 1-7.

Handy, S. L., Xing, Y., \& Buehler, T. J. (2010). Factors associated with bicycle ownership and use: A study of six small US cities. Transportation, 37(6), 967-985.

Hankey, S., \& Lindsey, G. (2016). Facility-demand models of peak-period pedestrian and bicycle traffic: A comparison of fully-specified and reduced-form models. Paper presented at the Transportation Research Board 95th Annual Meeting, Washington, DC.

Hankey, S., Lindsey, G., \& Marshall, J. (2014). Day-of-year scaling factors and design considerations for nonmotorized traffic monitoring programs. Transportation Research Record, 2468, 64-73.

Hankey, S., Lindsey, G., Wang, X., Borah, J., Hoff, K., Utecht, B., \& Xu, Z. (2012). Estimating use of non-motorized infrastructure: Models of bicycle and pedestrian traffic in Minneapolis, MN. Landscape and Urban Planning, 107(3), 307-316. doi: http://dx.doi.org/10.1016/j.landurbplan.2012.06.005

Heinen, E., van Wee, B., \& Maat, K. (2010). Commuting by bicycle: An overview of the literature. Transport Reviews, 30(1), 59-96.

Krizek, K. J., El-Geneidy, A., \& Thompson, K. (2007). A detailed analysis of how an urban trail system affects cyclists' travel. Transportation, 34(5), 611-624. doi: 10.1007/s11116-007-9130-z

Kuzmyak, J. R., Walters, J., Bradley, M., \& Kockelman, K. M. (2014). Estimating bicycling and walking for planning and project development: A guidebook. Washington, DC: Transportation Research Board.

Lindsey, G., Wilson, J., Yang, J. A., \& Alexa, C. (2008). Urban greenways, trail characteristics and trail use: implications for design. Journal of Urban Design, 13(1), 53-79.

Lovasi, G. S., Schwartz-Soicher, O., Neckerman, K. M., Konty, K., Kerker, B., Quinn, J., \& Rundle, A. (2013). Aesthetic amenities and safety hazards associated with walking and bicycling for transportation in New York City. Annals of Behavioral Medicine, 45(1), 76-85.

Ma, L., \& Dill, J. (2015). Associations between the objective and perceived built environment and bicycling for transportation. Journal of Transport \& Health, 2(2), 248-255.

Moran, M. R., Plaut, P., \& Epel, O. B. (2015). Do children walk where they bike? Exploring built environment correlates of children's walking and bicycling. Journal of Transport and Land Use, 9(2), 43-65.

Moudon, A. V., Lee, C., Cheadle, A. D., Collier, C. W., Johnson, D., Schmid, T. L., \& Weather, R. D. (2005). Cycling and the built environment, a US perspective. Transportation Research Part D: Transport and Environment, 10(3), 245-261. 
Muhs, C. D., \& Clifton, K. J. (2016). Do characteristics of walkable environments support bicycling? Toward a definition of bicycle-supported development. Journal of Transport and Land Use, 9(2), 147-148.

Niemeier, D. A. (1996). Longitudinal analysis of bicycle count variability: Results and modeling implications. Journal of Transportation Engineering, 122(3), 200-206.

Noland, R. B., Smart, M. J., \& Guo, Z. (2016). Bikeshare Trip Generation in New York City. Paper presented at the Transportation Research Board 95th Annual Meeting, Transportation Research Board, Washington, DC.

Pikora, T., Giles-Corti, B., Bull, F. , Jamrozik, K., \& Rob, D. (2003). Developing a framework for assessment of the environmental determinants of walking and cycling. Social Science and Medicine, 56(8), 1693-1703.

Pucher, J., Dill, J., \& Handy, S. (2010). Infrastructure, programs, and policies to increase bicycling: An international review. Preventive medicine, 50, S106-S125.

Qureshi, I., \& Fang, Y. (2010). Socialization in open source software projects: A growth mixture modeling approach. Organizational Research Methods, 4(1), 208-238.

Reynolds, C. C., Harris, M. A., Teschke, K., Cripton, P. A., \& Winters, M. (2009). The impact of transportation infrastructure on bicycling injuries and crashes: A review of the literature. Environmental Health, 8(1), 47.

Saelens, B., Sallis, J., \& Frank, L. (2003). Environmental correlates of walking and cycling: Findings from the transportation, urban design, and planning literatures. Annals of Behavioral Medicine, 25(2), 80-91. doi: 10.1207/s15324796abm2502_03

Schoner, J. E., \& Levinson, D. M. (2014). The missing link: Bicycle infrastructure networks and ridership in 74 US cities. Transportation, 41(6), 1187-1204.

Seattle Department of Transportation (2014). Seattle Master Bicycle Plan, Seattle Department of Transportation, Seattle.

Shaheen, S., Guzman, S., \& Zhang, H. (2010). Bikesharing in Europe, the Americas, and Asia: Past, present, and future. Transportation Research Record, 2143, 159-167.

Shoup, D. C. (2005). The high cost of free parking (Vol. 206). Chicago: Planners Press.

Stefansdottir, H. (2014). A theoretical perspective on how bicycle commuters might experience aesthetic features of urban space. Journal of Urban Design, 19(4), 496-510.

Strauss, J., \& Miranda-Moreno, L. (2013). Spatial modeling of bicycle activity at signalized intersections. The Journal of Transport and Land Use, 6(2), 47-58.

Stryhn, H., Sanchez, J., Morley, P., Booker, C., \& Dohoo, I. R. (2006). Interpretation of variance parameters in multilevel Poisson regression models. Proceedings of the 11th International Symposium on Veterinary Epidemiology and Economics, International Society for Veterinary Epidemiology and Economics, Cairns, Australia.

Su, M., Tan, Y.-Y., Liu, Q.-M., Ren, Y.-J., Kawachi, I., Li, L.-M., \& Lv, J. (2014). Association between perceived urban built environment attributes and leisure-time physical activity among adults in Hangzhou, China. Preventive Medicine, 66, 60-64.

Thomas, B., \& DeRobertis, M. (2013). The safety of urban cycle tracks: A review of the literature. Accident Analysis \& Prevention, 52(0), 219-227. doi: http://dx.doi.org/10.1016/j.aap.2012.12.017

Tin, S., Woodward, A., Robinson, E., \& Ameratunga, S. (2012). Temporal, seasonal and weather effects on cycle volume: An ecological study. Environmental Health, 11(1), 12.

Van Acker, V., Derudder, B., \& Witlox, F. (2013). Why people use their cars while the built environment imposes cycling. Journal of Transport and Land Use, 6(1), 53-62.

Vanparijs, J., Panis, L. I., Meeusen, R., \& de Geus, B. (2015). Exposure measurement in bicycle safety 
analysis: A review of the literature. Accident Analysis \& Prevention, 84, 9-19.

Wang, X., Lindsey, G., Schoner, J. E., \& Harrison, A. (2015). Modeling bike share station activity: Effects of nearby businesses and jobs on trips to and from stations. Journal of Urban Planning and Development, 142(1), [04015001]. doi: 10.1061/(ASCE)UP.1943-5444.0000273

Wardman, M., Tight, M., \& Page, M. (2007). Factors influencing the propensity to cycle to work. Transportation Research Part A: Policy and Practice, 41(4), 339-350. doi: http://dx.doi.org/10.1016/j. tra.2006.09.011

Winters, M., Davidson, G., Kao, D., \& Teschke, K. (2011). Motivators and deterrents of bicycling: Comparing influences on decisions to ride. Transportation, 38(1), 153-168.

Xing, Y., Handy, S. L., \& Mokhtarian, P. L. (2010). Factors associated with proportions and miles of bicycling for transportation and recreation in six small US cities. Transportation research part D: Transport and Environment, 15(2), 73-81. 